Nova Southeastern University

Florida

NOVA SOUTHEASTERN

UNIVERSITY

NSUWorks

Marine \& Environmental Sciences Faculty Articles Department of Marine and Environmental Sciences

6-1-2012

\title{
Open and Closed Seascapes: Where Does Habitat Patchiness Create Populations with High Fractions of Self-Recruitment?
}

\author{
Malin L. Pinsky \\ Princeton University
}

Stephen R. Palumbi

Stanford University

Serge Andrefouet

Institut de Recherche pour le Developpement - France

Samuel J. Purkis

Nova Southeastern University, purkis@nova.edu

Find out more information about Nova Southeastern University and the Halmos College of Natural Sciences and Oceanography.

Follow this and additional works at: https://nsuworks.nova.edu/occ_facarticles

Part of the Marine Biology Commons, and the Oceanography and Atmospheric Sciences and Meteorology Commons

\section{NSUWorks Citation}

Malin L. Pinsky, Stephen R. Palumbi, Serge Andrefouet, and Samuel J. Purkis. 2012. Open and Closed Seascapes: Where Does Habitat Patchiness Create Populations with High Fractions of Self-Recruitment? .Ecological Applications , (4) : 1257 -1267.

https://nsuworks.nova.edu/occ_facarticles/254. 


\title{
Open and closed seascapes: Where does habitat patchiness create populations with high fractions of self-recruitment?
}

\author{
Malin L. Pinsky,${ }^{1,5}$ Stephen R. Palumbi, ${ }^{2}$ Serge Andréfouët, ${ }^{3}$ and Sam J. Purkis ${ }^{4}$ \\ ${ }^{1}$ Department of Ecology and Evolutionary Biology, Princeton University, 106 A Guyot Hall, Princeton, New Jersey 08544 USA \\ ${ }^{2}$ Hopkins Marine Station, Department of Biology, Stanford University, 120 Oceanview Boulevard, \\ Pacific Grove, California 93950 USA \\ ${ }^{3}$ Institut de Recherche pour le Développement, Centre de Nouméa, B.P. A5, 98848, Nouméa, New Caledonia \\ ${ }^{4}$ National Coral Reef Institute, Nova Southeastern University Oceanographic Center, Dania Beach, Florida 33004 USA
}

\begin{abstract}
Which populations are replenished primarily by immigrants (open) and which by local production (closed) remains an important question for management with implications for response to exploitation, protection, and disturbance. However, we lack methods for predicting population openness. Here, we develop a model for openness and show that considering habitat isolation explains the existence of surprisingly closed populations in highdispersal species, including many marine organisms. Relatively closed populations are expected when patch spacing is more than twice the standard deviation of a species' dispersal kernel. In addition, natural scales of habitat patchiness on coral reefs are sufficient to create both largely open and largely closed populations. Contrary to some previous interpretations, largely closed marine populations do not require mean dispersal distances that are unusually short, even for species with relatively long pelagic larval durations. We predict that habitat patchiness has strong control over population openness for many marine and terrestrial species with a highly dispersive life stage and relatively sedentary adults. This information can be used to make initial predictions about where populations will be more or less resilient to local exploitation and disturbance.
\end{abstract}

Key words: connectivity; coral reef seascapes; dispersal; landscape ecology; marine protected areas; metapopulation; population openness; reef fishes.

\section{INTRODUCTION}

Ecologists frequently classify populations as open or closed, depending on whether they are replenished primarily by immigrants or by local production (Thomas and Kunin 1999, Hixon et al. 2002). Recruitment is directly related to local reproduction in closed populations, but independent in populations that are open. This distinction has important implications for management and affects a population's response to exploitation or protection, recovery from disturbance, degree of local adaptation, source of density regulation, interactions with other species, and population dynamics (Palmer et al. 1996, Roberts 1997, Armsworth 2002, Hixon et al. 2002, Lenormand 2002, Leibold et al. 2004, Wieters et al. 2008). For example, because immigrants will continue to arrive to an open population even if local adults become rare or extirpated, relatively open populations can recover quickly after disturbance or exploitation (Roberts 1997). In contrast, recruitment declines with adult abundance in closed populations.

An important question is therefore which populations are open, which closed, and which somewhere in

Manuscript received 9 July 2011; revised 30 January 2012; accepted 1 February 2012. Corresponding Editor: J. J. Vander Zanden.

${ }^{5}$ E-mail: pinsky@princeton.edu between. Many authors have suggested that we should expect open populations for species with a highly dispersive life stage, including many plants, winddispersed insects, stream-dwelling species, and marine organisms, particularly at narrow spatial scales (Palmer et al. 1996, Thomas and Kunin 1999, Hixon et al. 2002, Mora and Sale 2002). In contrast, we should expect closed populations at wide spatial scales and for species with discrete populations and little to no dispersal. This guidance, however, is only qualitative, and may lead to misinterpretation if not addressed quantitatively.

As defined in the literature, population openness is one minus the probability that an arriving recruit was born within the population in question (Hixon et al. 2002). This quantity has been attractive to researchers because it can be measured empirically. For example, a number of studies have measured relatively low openness in marine species, despite the fact that these species' larvae spend weeks to months dispersing and have the ability to travel long distances (Jones et al. 1999, 2005, Swearer et al. 1999, Taylor and Hellberg 2003, Almany et al. 2007). These observations have prompted a range of hypotheses for how largely closed populations can arise despite high dispersal abilities. Explanations include natural selection against immigrants, mortality of dispersing individuals, and physical retention mechanisms (Paris and Cowen 2004, Shanks 2009, Marshall 
et al. 2010, Shima et al. 2010). For many marine species, one widespread interpretation is that mean dispersal distances are much shorter than previously suspected (Warner and Cowen 2002, Levin 2006), and perhaps only hundreds of meters in species with relatively closed populations (Shanks 2009). The implicit assumption in many of these interpretations is that relatively closed populations must be evidence that mean dispersal distances are short. If true, one implication would be that protected areas developed for these species could be much smaller than previously suspected, since the required size for protected areas scales with the mean dispersal distance (Botsford et al. 2001, Lockwood et al. 2002).

What, however, is the relationship between dispersal abilities and population openness? Is a relatively closed population an accurate indication of short mean dispersal distances? To date, this question has been left to qualitative judgment and terms like high, low, local, and regional. We lack the quantitative theory to predict the level of population openness we should expect in a given situation and against which observations could be compared. A model would also facilitate increased communication among empiricists measuring population openness and theoreticians examining spatial population dynamics, as has been called for by previous authors (Kinlan et al. 2005, Botsford et al. 2009).

In this paper, we develop a simple model for population openness that is appropriate for species with sedentary adults and dispersing juveniles (e.g., a seed or larval stage). We use coral reef fishes as an illustrative example because recent empirical papers have highlighted what appear to be surprisingly closed populations. Our model considers dispersal ability as well as habitat patchiness. This latter addition facilitates the application of our model to realistic landscapes and seascapes, and, as we will show, has important impacts on openness. Most habitats exist as patches in a less suitable matrix, including meadows, forests, mountaintops, and estuaries (Saunders et al. 1991, Andréfouët et al. 2006). Habitats are also highly patchy in the sea, including kelp forests, rocky shores, coral and rocky reefs, sheltered bays and estuaries, and deep-sea vents (Kritzer and Sale 2006). Biophysical models of marine larval dispersal have suggested that large and isolated habitat patches can have relatively closed populations (James et al. 2002, Cowen et al. 2006), and recent empirical studies have found that larval connectivity among coral reef patches declines with distance (Buston et al. 2011, SaenzAgudelo et al. 2011). Previous authors have also suggested that geographic setting may be an important driver of population-level processes in marine fishes (Jones et al. 2009), but it remains unclear whether (or where) natural scales of habitat patchiness are sufficient to have an impact on openness. In general, patchiness is unimportant if dispersal easily crosses habitat gaps (Wiens 1989). To date, however, we lack quantitative comparisons between dispersal distances and empirical scales of habitat patchiness throughout the ocean.

Renewed attention to habitat patchiness is also timely given the strong focus on patchiness in metapopulation and landscape theory, including the effects of matrix quality and patch number, spacing, and quality on population persistence and equilibrium density (e.g., Bascompte et al. 2002, Hastings and Botsford 2006, Moilanen and Hanski 2006, Figueira 2009, Kaplan et al. 2009, Shima et al. 2010). Similarly, protected area and marine reserve theory have examined how population survival depends on reserve size and spacing (Botsford et al. 2001, Drechsler et al. 2003, Gaines et al. 2010, White et al. 2010). In this body of theory, however, the focus has largely been on understanding how patches contribute to regional persistence at a metapopulation level.

In contrast, our paper aims to bridge the gap between theory and empirical research by focusing on a quantity (openness) that is commonly measured in the field. As mentioned earlier in the Introduction, openness is also interesting from a population dynamics perspective because it indicates where regional processes (through immigration) have important impacts on local dynamics, including recovery from disturbance. From a local manager's perspective, population openness determines whether local overharvest or conservation will have direct consequences for local persistence, or whether activities elsewhere that affect immigration will be a more important consideration. So far, however, a quantitative treatment of population openness has been overlooked.

The goal of this paper, therefore, is to determine the conditions under which habitat patchiness can create closed populations and ask whether these conditions are likely to be common in marine ecosystems. To do so, we first develop a simple model to connect habitat patchiness and dispersal ability to the degree of population openness. We then examine whether previous empirical studies are likely to fit the conditions for relatively closed populations. Finally, we ask whether such conditions are likely to be common in the natural world by using a variety of coral reef seascapes mapped by remote sensing. We argue that studies measuring selfrecruitment or population openness must be interpreted in the context of habitat patchiness. Some observations of relatively closed marine populations may result from previously underappreciated impacts of habitat patchiness on the source of larvae, rather than from short mean dispersal distances.

\section{Materials and Methods}

\section{Model}

To connect habitat patchiness to population openness, we started from a simple connectivity metric inspired by metapopulation theory (Moilanen and Hanski 2006). We calculated the number of immigrants $\left(I_{i}\right)$ into patch $i$ : 

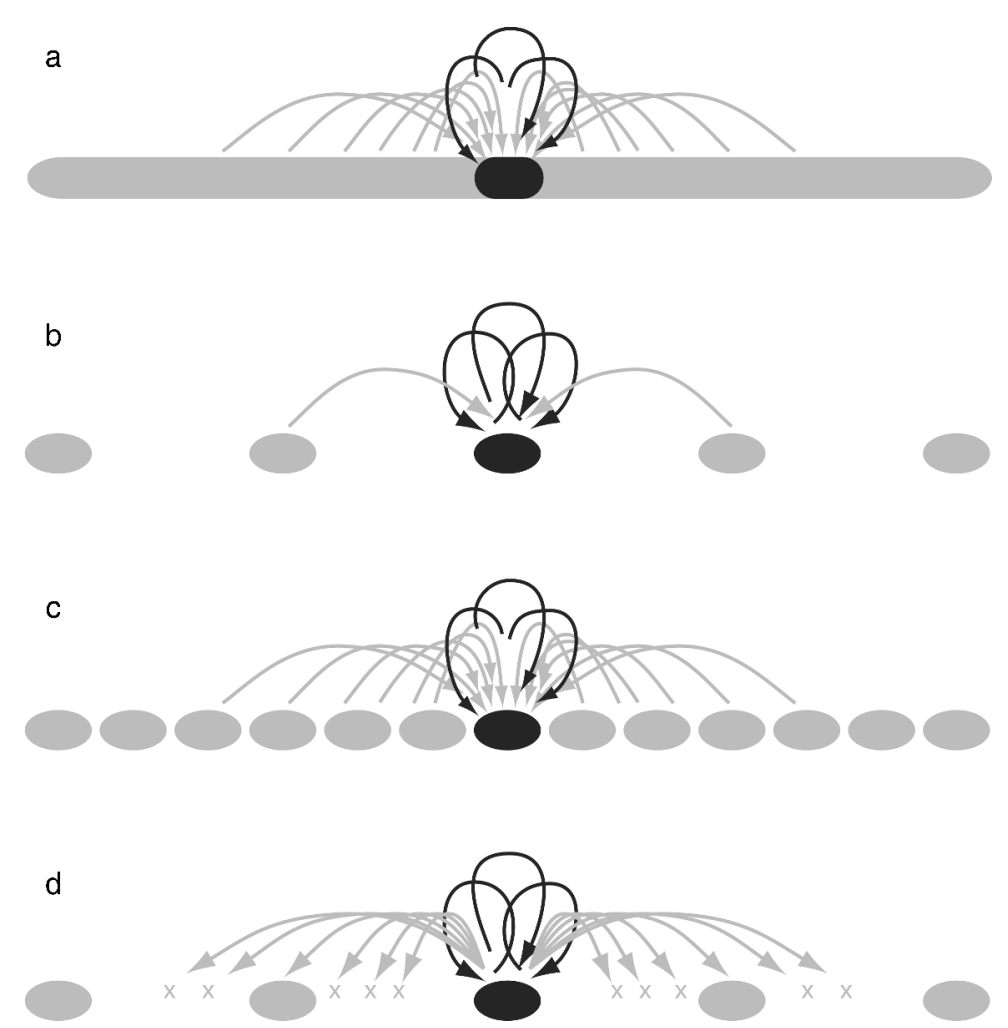

FIG. 1. Habitats can be (a) continuous or (b-d) patchy. (a) In continuous habitats, large numbers of immigrants into the focal population (gray arrows) create a population that is largely open. (b) In patchy environments, fewer immigrants reach the focal population, and the population is therefore more closed. (c) If the spacing between patches is small relative to dispersal abilities, the openness of the population will not be affected by habitat patchiness (compare to a). (d) The population will have low retention if most larvae disperse away from the patch, and this can easily occur even in relatively closed populations if habitats are patchy (compare to b).

$$
I_{i}=\sum_{j \neq i} D_{j, i} N_{j}
$$

where $D_{j, i}$ was the probability of a larva from patch $j$ settling on patch $i$, and $N_{j}$ was the number of offspring produced by patch $j$. The dispersal kernel $(D)$ was a probability density function describing the distribution of dispersed larvae, but did not consider the availability of habitat at settlement locations (see Botsford et al. 2009). Following previous convention, we included any mortality that occurs during dispersal in the dispersal kernel $(D)$, and so $D$ sums to much less than one for most marine species with high larval mortality (Botsford et al. 2009). A kernel summarizes the impacts of many "biological barriers" that occur during dispersal, including predation and starvation that are related to time in the plankton or distance (Marshall et al. 2010), but does not include any post-settlement mortality that might favor (Hamilton et al. 2008) or select against (Marshall et al. 2010) immigrants. We defined the kernel at settlement because most empirical measurements of openness are made at or quite near the time of settlement rather than substantially later. Empirical deviations from our model can suggest where such processes may be important, however.
We next used the same logic to define the number of individuals that return to the patch in which they were born:

$$
R_{i}=D_{i, i} N_{i}
$$

where $D_{i, i}$ was the probability of returning to patch $i$. We used Eqs. 1 and 2 to define openness $(S)$, which is the fraction of settling individuals that are immigrants (Fig. $1 \mathrm{a}-\mathrm{c})$ :

$$
S_{i}=\frac{I_{i}}{I_{i}+R_{i}}
$$

where $R_{i}$ and $I_{i}$ were the numbers of self-recruiting individuals and the number of immigrants (respectively), as defined in Eqs. 1 and 2. This equation directly measured population openness as it has been defined previously (Hixon et al. 2002). Because $I$ was sensitive to the location of habitat patches and the distances between them, $S$ was also sensitive to seascape geometry.

We note that openness is simply one minus selfrecruitment, where self-recruitment is the probability that an arriving recruit was born within the local population (Botsford et al. 2009). The two therefore measure the same thing, but on a different scale, and we 
use both throughout our paper to reflect the use of both terms in the scientific literature. Self-recruitment is commonly reported by empirical studies of marine larval dispersal (Jones et al. 1999, 2005, Swearer et al. 1999, Almany et al. 2007).

We also note that openness (or self-recruitment) has often been confused with retention (e.g., Kinlan et al. 2005), despite their substantial differences. Retention measures the proportion of larvae produced in a local population that settles in that population:

$$
T_{i}=\frac{R_{i}}{N_{i}}=D_{i, i}
$$

The key difference between openness (or self-recruitment) and retention is that the former indicates the source of locally settling individuals (Fig. 1a-c), while the latter specifies the destination of locally produced individuals (Fig. 1d). The distinction lies in the denominator; while openness (or self-recruitment) is calculated as a fraction of all recruiting individuals $\left(S_{i}+\right.$ $R_{i}$ ), retention is calculated as a fraction of all locally produced individuals $(N)$. For a helpful discussion of these issues, see Botsford et al. (2009).

\section{Simplifications for applying the model}

To implement this model, we calculated openness and retention using simple approximations for $D_{j, i}$ and $N_{j}$. We used a normal dispersal kernel, a form that arises both from a random walk dispersal process (Skellam 1951) and from averaging across many quasi-random larval trajectories in a coastal ocean (Siegel et al. 2008). Dispersal ability was determined by the standard deviation of this kernel $(\sigma)$, which is called dispersal spread (Siegel et al. 2003). Mean dispersal distance for a 1D (one-dimensional) normal kernel can be calculated as $\sigma \sqrt{2 / \pi}$.

We centered the dispersal kernel on zero as our base scenario, but we investigated the effects of directional dispersal (e.g., advection by currents) by offsetting the kernel from zero by a mean displacement. We scaled advection so that it increased proportionally with dispersal spread. This ensured that the ratio of the two (called the Peclet number) remained constant, as has been suggested before (Gaylord and Gaines 2000, White et al. 2010).

We used a regular grid for our patches to ensure that we measured openness at a consistent spatial scale. Because all patches therefore had the same area, we also assumed that larval production was the same in each patch. Both the dispersal kernel and larval production approximations could be replaced if oceanographic models of connectivity (e.g., Cowen et al. 2006, Siegel et al. 2008) or metrics of local production (e.g., Watson et al. 2010) were available.

The net effect of these simplifications was that openness became a function of dispersal spread and patch geometry. This allowed us to calculate dispersal spread if patch geometry and openness were known.

\section{Application to simple seascapes}

To explore our model, we applied it first to highly simplified "dashed line" coastlines. The dashed line was similar to many marine reserve models (e.g., Botsford et al. 2001) and consisted of an infinite, 1D array of 500-m habitat patches separated by uninhabitable spaces. We also investigated a 2D (two-dimensional) grid of habitat patches. Spacing between adjacent patches was measured from patch center to patch center. A halfkilometer patch width was arbitrary, but allowed comparison to previous field studies. This scale does not necessarily correspond to the spatial scale of ecological or evolutionary populations, as these can vary dramatically in size depending on the definition used (Waples and Gaggiotti 2006).

\section{Application to remotely sensed seascapes}

To determine whether closed populations are likely to be common, we analyzed 17 coral reef seascapes. These seascapes were previously classified from 30-m spatial resolution Landsat satellite images (Andréfouët et al. 2006, Wabnitz et al. 2010). The individual seascapes (Appendix: Fig. A1) were chosen to represent the global diversity of coral reefs. For analysis, the reefs were converted to a $500 \times 500 \mathrm{~m}$ grid. Grid cells were specified as suitable habitat if $>50 \%$ of the cell was covered by coral reef. We calculated $S$ for each grid cell with Eq. 3 and a two-dimensional, symmetrical, normal dispersal kernel. Because few patches were isolated by land (see Appendix: Fig. A1), we did not prevent larvae from dispersing across land.

\section{RESUlts}

\section{Population openness in simple seascapes}

We first tested our model on a uniform line of habitat patches (Fig. 1b). In this context, $S$ varied from nearly $0 \%$ to nearly $100 \%$, depending on the relative values of dispersal spread and habitat spacing (Fig. 2). As expected, high spacing between habitat patches and short dispersal created closed patches with low immigration (lower right of Fig. 2), while the opposite created open patches (upper left of Fig. 2). Changing the scale of analysis (different patch size) had negligible impact on this graph because all patches in this simple model were the same size. Choosing a larger size created patches with both more local recruits and more immigrants, but effectively the same openness. In two dimensions (Fig. 2b), patches tended to be somewhat more open for the same patch spacing and dispersal spread because there were a greater number of surrounding patches to contribute immigrants. Patches were also more open in advective environments because fewer larvae returned to the patch from which they were born (Appendix: Fig. A2).

As a general guideline, our model predicted relatively closed patches if spacing was more than about twice the dispersal spread (diagonal of Fig. 2). For a leptokurtic kernel (fat tails and high central peak), patches become 
closed at narrower spacing, and so this guideline tends to be conservative. In advective environments, wider spacing was required for closed populations (Appendix: Fig. A2).

An example illustrates the important difference between openness and retention. For a species with 10$\mathrm{km}$ dispersal spread in a habitat with $25-\mathrm{km}$ gaps between patches, patches had both low retention $(>98 \%$ of larvae emigrated) and low openness (only $8 \%$ of settling larvae were immigrants). Put another way, the patches had both low retention and high self-recruitment (92\% of settling larvae were from local parents). This situation corresponds to the lower right of Fig. 2.

When habitat spacing was about twice the dispersal spread, small differences in dispersal or spacing had relatively large effects on $S$ (along the diagonal of Fig. 2). If marine environments are patchy at scales similar to an organism's dispersal ability, seascapes should contain both relatively open and relatively closed patches (high and low $S$, respectively).

\section{Comparison to empirical studies}

It appears that many recent marine larval tagging studies have been conducted in the zone where both open and closed populations are likely, and particular care should be used when interpreting their results. For example, three studies observed openness of $40-70 \%$ (self-recruitment of $60-30 \%$ ) to $\sim 500$-m habitat patches in four species of coral reef fish (genera Amphiprion and Chaetodon; Jones et al. 2005, Almany et al. 2007, Planes et al. 2009). An important but easily overlooked aspect of these studies is that the nearest habitat patch was 5$20 \mathrm{~km}$ away. Using the range of patch spacing and openness reported by these studies, we delineated their parameter space with the dashed polygon in Fig. 2 ( $x$ axis, 5-20 km; $y$-axis, $40-70 \%$ openness).

While none of these studies measured dispersal spread, our model allowed us to infer what it might be. For example, along a $1 \mathrm{D}$ coastline with $10-\mathrm{km}$ patch spacing, our model suggested that a dispersal spread of $12 \mathrm{~km}$ would be most compatible with $70 \%$ openness (or $30 \%$ selfrecruitment; e.g., Jones et al. [2005] studying Amphiprion polymnus). In a $2 \mathrm{D}$ ocean with $20-\mathrm{km}$ patch spacing, a dispersal spread of $10 \mathrm{~km}$ would be most compatible with $40 \%$ openness (or $60 \%$ self-recruitment; e.g., Almany et al. [2007] studying Amphiprion percula and Chaetodon vagabundus). In a $2 \mathrm{D}$ ocean with only $5-\mathrm{km}$ spacing, however, our model needed only a $2.5-\mathrm{km}$ dispersal spread to produce $40 \%$ openness. In all cases, our results suggested that the three empirical studies would have observed dramatically more closed populations if dispersal spread in these coral reef fishes was $<2 \mathrm{~km}$.

\section{Closed populations within empirical seascapes}

Upon examining empirical seascapes, we found that they had both open and relatively closed patches. We first show examples from the Bahamas with 2-km dispersal spread and from Papua New Guinea with 5$\mathrm{km}$ dispersal (Fig. 3). These examples were chosen to

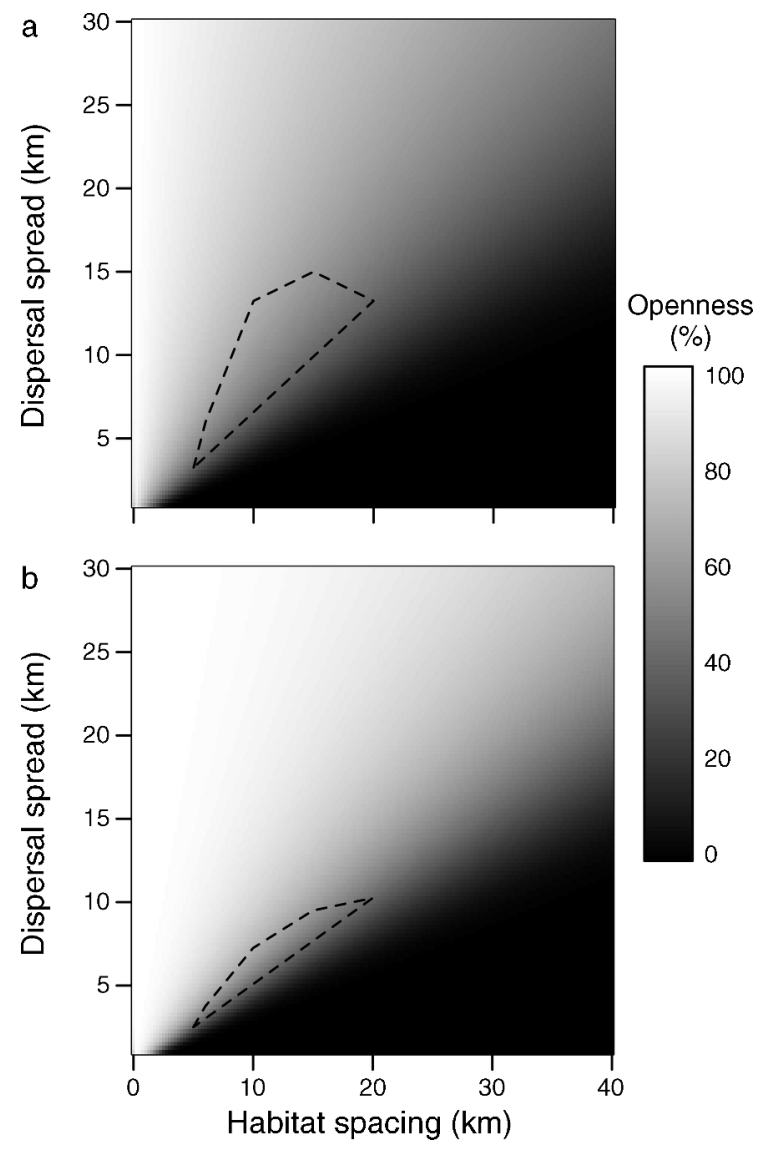

FIG. 2. Openness $(S)$ of patches predicted from a normal dispersal kernel in a patchy habitat that was (a) a onedimensional coastline (similar to Fig. 1b) or (b) a twodimensional grid. Patches were $500 \mathrm{~m}$ wide, and spacing measured the distance between patch centers. When spacing between patches was more than about twice the dispersal spread, $S$ was low and percentage self-recruitment was high (lower right). The dashed polygon outlines the ranges of habitat spacing and values of $S$ measured by three marine population openness studies (Jones et al. 1999, Almany et al. 2007, Planes et al. 2009).

illustrate the range of $S$ predicted within each seascape. In both, the patches embedded in continuous sections of reef had high $S$ (up to $98 \%$ ) and would be classified as open at this scale. In addition, a small number of patches were more isolated and had $S$ as low as $44 \%$. These latter patches received up to $56 \%$ of their recruits from local parents (56\% self-recruitment) and would appear quite closed.

We next applied our models across 17 coral reef seascapes (Appendix: Fig. A1) to ask whether all seascapes are likely to contain closed populations. We found surprising similarities among seascapes (Fig. 4). While seascapes on average were open across all dispersal spreads (Fig. 4a), we also found that all seascapes contained at least some isolated patches that were largely closed and had low $S$ (Fig. 4b). All seascapes exhibited a similar relationship between 

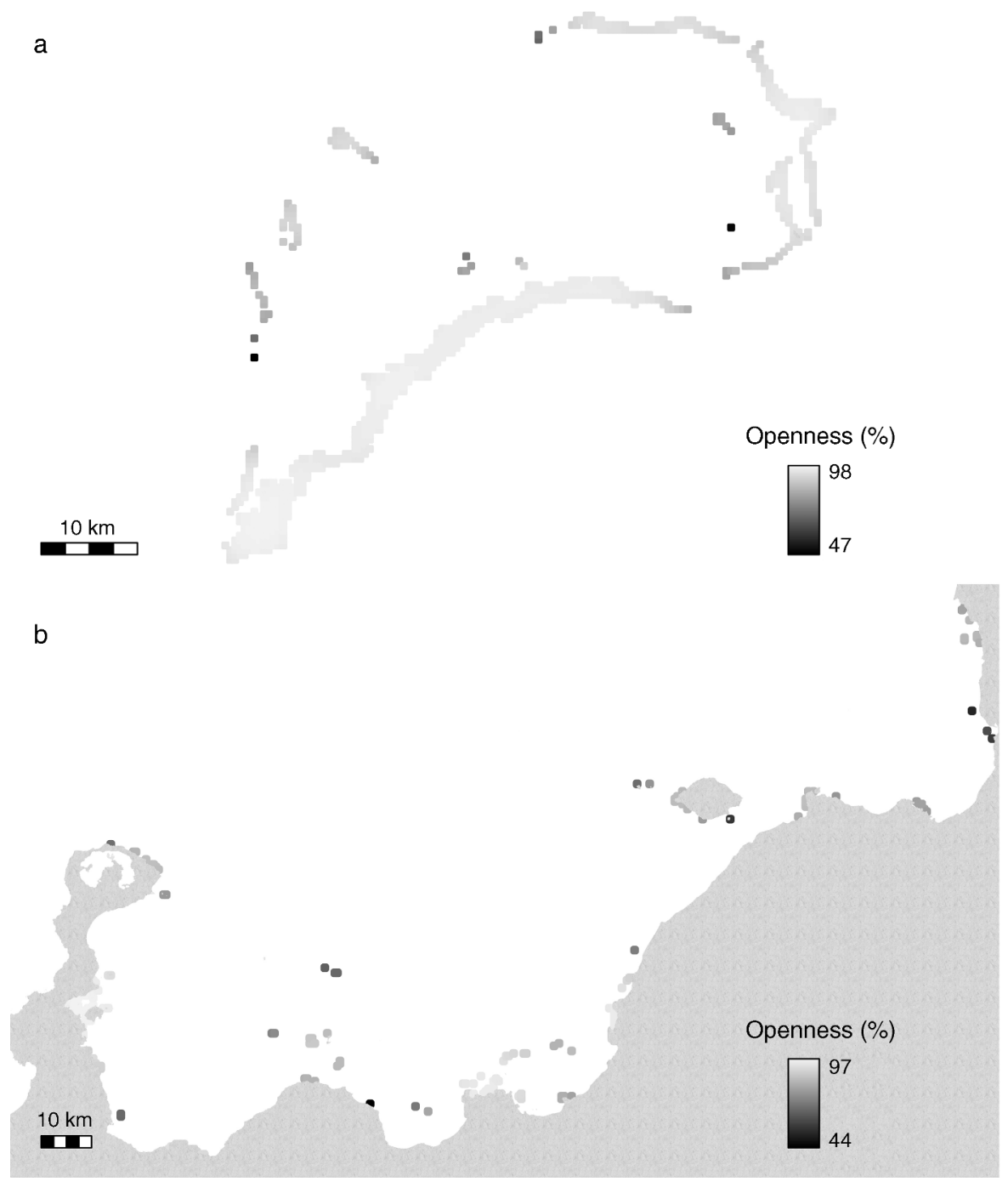

FIG. 3. Variation in openness $(S)$ across patchy seascapes: (a) Ragged Island, Bahamas (2-km dispersal spread) and (b) Kimbe Bay, Papua New Guinea (5-km dispersal spread). Grid cells were 500-m squares, and the dispersal kernel was 2-D (twodimensional) normal. Each seascape had a large number of well-connected reefs with high immigration and a smaller number of relatively closed populations with as low as $44 \%$ openness ( $56 \%$ self-recruitment). The textured gray fill in panel (b) indicates land.

openness and minimum dispersal spread, with a threshold near $10 \mathrm{~km}$ (Fig. 4b). Closed patches were most likely for species with dispersal spread $<5 \mathrm{~km}$.

Substantial variation between seascapes was also apparent. On continuous reefs such as Northwest Belep (New Caledonia 2), patches were generally open even at short dispersal spreads. In highly patchy seascapes such as Kimbe Bay (Papua New Guinea 3), some patches were moderately closed even for dispersal spreads up to $10 \mathrm{~km}$. The somewhat unique curve in the Java Sea (Indonesia 1) was created because two adjacent patches were quite isolated and therefore had a predicted $S$ near $50 \%$ for dispersal spreads of $2-5 \mathrm{~km}$ (Fig. 4b). In all seascapes, however, all patches were open for species with dispersal spread $>20 \mathrm{~km}$.
Average nearest neighbor distance was a reasonable predictor of average openness $(S)$ within seascapes (Fig. 5; $\left.P<0.003, r^{2}>46 \%\right)$. Seascapes with wider spacing had lower $S$, and the populations in these patchier seascapes were more closed.

Finally, we note that we conducted our analyses at only a single grid scale to illustrate a general pattern across many seascapes. Following the logic of our models, however, analyses with a larger grid size (e.g., 1 $\times 1 \mathrm{~km}$ ) generally showed populations that were more closed (Appendix: Fig. A3). Similarly, a smaller grid size showed populations that were more open.

\section{DisCUSSION}

By examining a simple model, we determined conditions under which habitat patchiness can have strong 

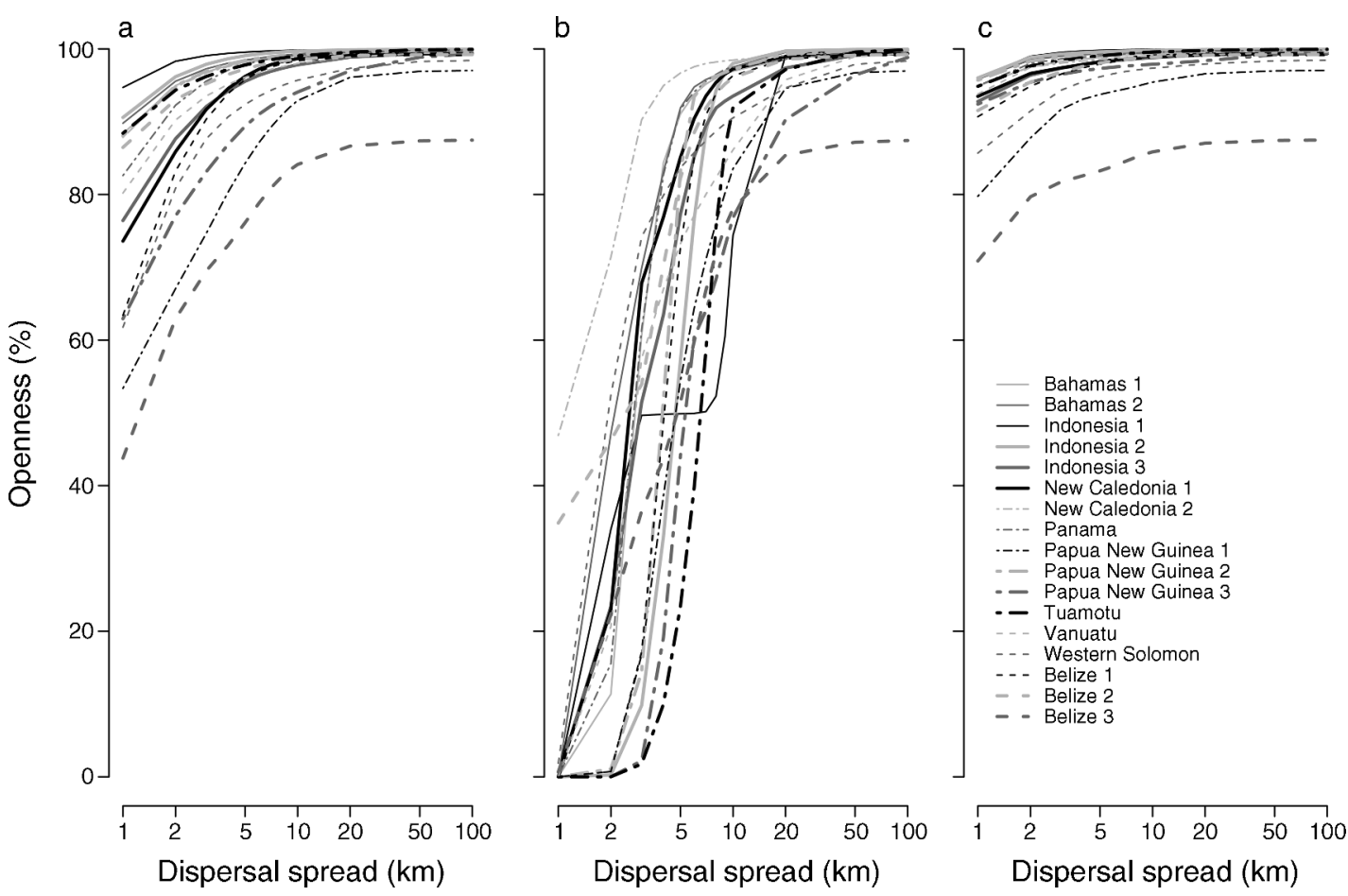

FIG. 4. Patch openness $(S)$ within each of 17 coral reef seascapes, as related to dispersal spread (note log scale): (a) mean, (b) minimum, and (c) maximum were calculated across all grid cells within a seascape. Minimum and maximum openness indicates the most or least closed habitat patch (respectively) in each seascape. Each line represents one seascape. Openness was calculated to 500 $\times 500 \mathrm{~m}$ grid cells. See Appendix: Fig. A1 for maps and locations of each seascape.

impacts on immigration and population openness. We found that relatively closed populations with few immigrants were more common where patch spacing was more than twice the dispersal spread. When investigating realistic levels of habitat patchiness, we found that a wide range of coral reef seascapes were likely to contain a mixture of isolated, relatively closed populations with low immigration and open populations with substantially more immigration. We found that patch spacing could explain why a number of recent studies have found surprisingly closed populations in marine fishes otherwise characterized by relatively wide dispersal kernels.

Our criteria for closed populations provide quantitative guidance on an issue that has been discussed qualitatively for many years (Hixon et al. 2002, Mora and Sale 2002, Warner and Cowen 2002). Our models of patchy habitats indicated that largely closed populations could arise for many species even at commonly encountered levels of patch isolation. Where a patch is isolated by more than twice the dispersal spread, relatively few individuals will immigrate from other patches and we should expect the population to be relatively closed. This criterion should be relevant not only to sedentary marine species with larval dispersal, but also to wind-dispersed insects, stream-dwelling aquatic organisms, plants with wind-dispersed seeds, and other organisms with a juvenile stage that disperses substantially farther than its adults.

We also note that our two-times rule for closed populations should not be confused with previous criteria for self-persistent populations. Theory suggests that isolated populations are more likely to survive on

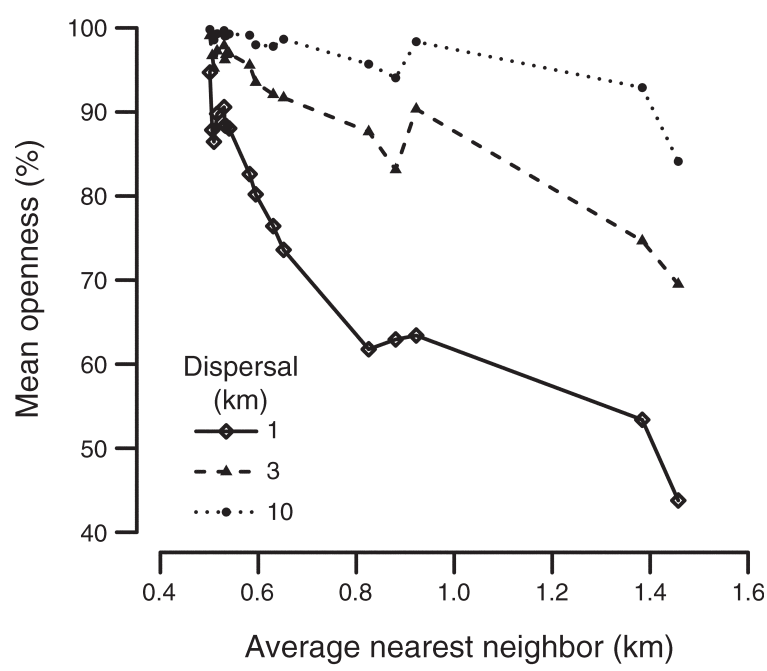

Fig. 5. Mean openness $(S)$ across $500 \times 500 \mathrm{~m}$ grid cells plotted against mean nearest-neighbor distance within each seascape. Each point is one of the 17 seascapes analyzed in this paper. 
patches at least twice as wide as mean dispersal distance (Botsford et al. 2001, Lockwood et al. 2002, White et al. 2010). Under such conditions, the fraction of larvae retained on the patch is high enough that the population can be sustained on local production alone. As a result, self-persistence depends on the relation between patch size and dispersal. In contrast, population openness depends on patch spacing and dispersal.

\section{Interpretation of empirical studies}

Appreciating the difference between persistence and openness is especially important for interpreting recent studies investigating the source of larvae recruiting to populations (e.g., Jones et al. 1999, 2005, Swearer et al. 1999, Almany et al. 2007). For example, it would be incorrect to assume that dispersal spread must be $<500$ $\mathrm{m}$ to explain relatively closed populations of reef fish on 500-m habitat patches (e.g., Shanks 2009). If dispersal spread was $<500 \mathrm{~m}$, these populations should have had virtually $0 \%$ openness, rather than the $40-70 \%$ openness observed. Instead, our model for population openness provided a quantitative method for interpreting these studies, and suggested that dispersal spread was one to two orders of magnitude greater $(3-15 \mathrm{~km}$, depending on the particular species and study). These numbers should be interpreted carefully because they rely on the assumption of a normal dispersal kernel and were based upon simple "dashed-line" or regular grid representations of habitat. However, recent field studies suggest that our model results are reasonable, despite its substantial simplifications. Population genetic methods suggest a dispersal spread near $10 \mathrm{~km}$ in other Amphiprion species (Pinsky et al. 2010, Saenz-Agudelo et al. 2011) and in other reef fish (Puebla et al. 2009).

One hypothesis stemming from our estimates of dispersal spread is that the above small island populations appear unlikely to persist on local reproduction alone, and therefore appear to require immigration for survival. We propose this hypothesis because the patch size of these populations appears to be substantially less than twice the mean dispersal distance, which is the standard minimum threshold for self-persistence (Lockwood et al. 2002). This self-persistence criterion should apply even though relatively high levels of self-recruitment have also been observed in these populations. Further work will be needed to test this hypothesis. Teasing apart the demographic consequences of dispersal (e.g., Carson et al. 2011) will help reveal how reef fish survive on small habitat patches and will have important implications for the design of marine protected areas (MPAs) and networks of MPAs.

\section{Openness in naturally patchy landscapes}

By analyzing a broad range of coral reef seascapes, we showed that natural variation in patch spacing is sufficient to have strong control over immigration rates, determining both whether and where populations are more open or closed. It has long been clear that extremely isolated islands harbor closed populations with little immigration (Robertson 2001), but it has not been clear that habitats are also sufficiently patchy at scales of tens of kilometers to create largely closed populations. Our results indicated that across 17 coral reef seascapes, populations with little immigration were always present, particularly for species with dispersal spread $<10 \mathrm{~km}$. Our maps provide initial hypotheses for where openness should be higher or lower, and these hypotheses can be tested with field observations. Where possible, a valuable extension to these models would be to use oceanographic currents to inform dispersal kernels and field surveys to guide larval production on each patch.

More generally, our analysis showed that the location of a population is important for determining how that population is replenished. A habitat patch being managed within a continuous string of other patches is likely to have high immigration in which larval supply or recruitment is independent of local production. In contrast, managers considering isolated habitat patches may encounter relatively closed populations where local production has a strong impact on larval supply. This realization also adds habitat configuration as an important aspect to consider in debates about the sources of regulation and density dependence in populations (Hixon et al. 2002). Models assuming open population dynamics (e.g., Bascompte et al. 2002) will be more appropriate in relatively continuous habitats, while models assuming partially closed dynamics (e.g., Bolker and Pacala 1999) will be appropriate in more isolated patches.

We chose to focus on coral reefs in this paper because there are readily available global data on their distribution, but we expect that our results will be broadly relevant across many habitats, both marine and terrestrial. While the patchiness of terrestrial habitats have long been recognized (Saunders et al. 1991), we also emphasize that many marine habitats are patchy as well, including estuaries, rocky reefs, deep sea vents, seamounts, rocky intertidal habitats, kelp forests, mangroves, seagrasses, tide pools, and sheltered bays (Kritzer and Sale 2006). Many marine species specialize on a single or small number of these habitat types as adults, and hence many species have patchy, fragmented distributions. We therefore predict that these species will have a range of both open and more closed populations. New efforts to map marine habitats, including with remote sensing, will be an important step toward understanding the role of patchiness in local demography (Andréfouët et al. 2006, Purkis et al. 2007).

As may have become clear, immigration fractions and population openness are matters of scale (Wiens 1989, Levin 1992). As other authors have noted, picking a wider spatial scale for investigation will reveal more strongly closed populations, while at a finer scale (e.g., a single coral head), populations will be almost entirely open (Wiens 1989, Hixon et al. 2002). Our chosen grid scale made our results relevant to previous studies, but 
higher spatial resolution remote sensing products (e.g., $30 \mathrm{~m}$ ) are available to resolve small reefs that may be important stepping stones for low dispersal species (Andréfouët et al. 2006). Temporal scale is also an important consideration, and our focus in this paper has been on single-generation, ecological time scales. Over evolutionary time scales of many generations, however, rare long distance dispersal events and multigenerational dispersal across intermediate stepping stones can keep populations evolutionarily connected even if they are ecologically quite closed (Waples 1998).

\section{Model limitations}

Our model represents the interactions of larval transport, behavior, and survival as a dispersal kernel that applies to all patches, and it captures the locations of larval production and settlement with a habitat map. These are clearly simplifications of marine dispersal, but our model's complexity is comparable to many useful metapopulation models (Moilanen and Hanski 2006). Updated versions of our model that use ocean currents to derive site-specific dispersal kernels would add accuracy to our predictions, but would not change our general conclusion that both relatively open and closed patches are likely to coexist within seascapes. Oceanographic features such as jets and gyres can create consistent places that favor transport or retention of larvae (Cowen et al. 2006), while heterogeneities in water quality can alter larval survival and affect population openness (Shima et al. 2010). Larval transport is also temporally stochastic, which means that managers cannot rely on recruitment in any single season to match results from a long-term average such as predicted in our model (White et al. 2011). Recovery after disturbance, for example, could be delayed or quickened by this stochasticity.

Our model could also be extended to consider other aspects of biology. For example, we assumed that population density was constant in our model application. When density varies, patches with higher density will experience more self-recruitment (lower openness) because they contribute proportionally more to the larval pool. It is a simple task to include variation in density when it is known by using location-specific values for $N$ (reproduction per patch). Certain types of larval behavior, such as homing to isolated islands, may also concentrate larvae near habitat patches (Leis et al. 2011), which would violate our assumption that the kernel is independent of habitat configuration. This behavior, though, will only decrease openness if larvae show stronger homing to their natal reef than to other reefs.

\section{Future directions}

Moving forward, it is interesting to note that conditions for persistence typically depend on retention or full dispersal matrices (Botsford et al. 2001, Lockwood et al. 2002, Byers and Pringle 2006, Hastings and Botsford 2006), while empirical studies measure open- ness or dispersal distance. This difference can make it difficult to translate empirical research into concrete advice for population management. Dispersal kernels, as used in this study, provide one method for translating between openness (or self-recruitment), dispersal spread, retention, and dispersal matrices. In fact, we demonstrated above how knowledge of seascape geography and openness can be used to infer dispersal spread under certain assumptions. If dispersal spread and shape are known (or assumed), they can be used to build simple dispersal matrices, though more research is needed into the temporal and spatial variation in kernels. More broadly, efforts to estimate dispersal spread and the shapes of dispersal kernels will help further the management and conservation of metapopulations.

Existing methods for estimating dispersal spread include isolation-by-distance genetic methods (Puebla et al. 2009, Pinsky et al. 2010), invasion rate estimates (Shanks 2009), and mark-recapture studies or their recent variations with natural and artificial tags (Jones et al. 1999, 2005, Swearer et al. 1999, Planes et al. 2009). All of these methods come with caveats, however. Isolation-by-distance methods require knowledge of genetic effective density, which remains difficult to estimate empirically. Invasion rates are often dominated by rare long-distance dispersal events, and can therefore overestimate average dispersal distance (Higgins and Richardson 1999). Mark-recapture experiments tend to underestimate average dispersal because short-distance dispersers are easier to find (Koenig et al. 1996). Coupled biophysical models provide impressively detailed spatial and temporal information on dispersal, though verifying the assumptions in these models remains challenging (James et al. 2002, Cowen et al. 2006, Watson et al. 2011). Further efforts to synthesize and compare multiple approaches will be very useful.

In conclusion, we argue that natural scales of habitat patchiness are likely to have strong impacts on population openness and self-recruitment for marine species. Seascape geography likely has a larger and more easily detectable role in determining population openness than has been appreciated to date, particularly for species with mean dispersal less than a few tens of kilometers. For these species, habitat patches with largely closed populations are likely to be quite common, at least on coral reefs. Managers considering how populations will respond to disturbance or exploitation can therefore use this seascape geography to inform their decisions.

\section{ACKNOWLEDGments}

We thank S. Gaines and F. Micheli for helpful conversations during this project and $\mathrm{G}$. de Leo for comments on an earlier draft. M. L. Pinsky was supported by an NSF Graduate Research Fellowship.

\section{Literature Cited}

Almany, G. R., M. L. Berumen, S. R. Thorrold, S. Planes, and G. P. Jones. 2007. Local replenishment of coral reef fish populations in a marine reserve. Science $316: 742-744$. 
Andréfouët, S., F. E. Muller-Karger, J. A. Robinson, C. J Kranenburg, D. Torres-Pulliza, S. A. Spraggins, and B. Murch. 2006. Global assessment of modern coral reef extent and diversity for regional science and management applications: a view from space. Pages 1732-1745 in Proceedings of the 10th International Coral Reef Symposium (Okinawa, Japan, 28 June-2 July 2004). Japanese Coral Reef Society, Tokyo, Japan.

Armsworth, P. R. 2002. Recruitment limitation, population regulation, and larval connectivity in reef fish metapopulations. Ecology 83:1092-1104.

Bascompte, J., H. P. Possingham, and J. D. Roughgarden. 2002. Patchy populations in stochastic environments: critical number of patches for persistence. American Naturalist 159:128-137.

Bolker, B. M., and S. W. Pacala. 1999. Spatial moment equations for plant competition: and the spatial strategies understanding advantages of short dispersal. American Naturalist 153:575-602.

Botsford, L. W., A. Hastings, and S. D. Gaines. 2001. Dependence of sustainability on the configuration of marine reserves and larval dispersal distance. Ecology Letters 4:144150.

Botsford, L. W., J. W. White, M.-A. Coffroth, C. B. Paris, S. Planes, T. L. Shearer, S. R. Thorrold, and G. P. Jones. 2009. Connectivity and resilience of coral reef metapopulations in marine protected areas: matching empirical efforts to predictive needs. Coral Reefs 28:327-337.

Buston, P. M., G. P. Jones, S. Planes, and S. R. Thorrold. 2011. Probability of successful larval dispersal declines fivefold over $1 \mathrm{~km}$ in a coral reef fish. Proceedings of the Royal Society B. http://dx.doi:10.1098/rspb.2011.2041

Byers, J. E., and J. M. Pringle. 2006. Going against the flow: retention, range limits and invasions in advective environments. Marine Ecology Progress Series 313:27-41.

Carson, H. S., G. S. Cook, P. C. López-Duarte, and L. A. Levin. 2011. Evaluating the importance of demographic connectivity in a marine metapopulation. Ecology 92:1972-1984.

Cowen, R. K., C. B. Paris, and A. Srinivasan. 2006. Scaling of connectivity in marine populations. Science 311:522-527.

Drechsler, M., K. Frank, I. Hanski, R. B. O'Hara, and C. Wissel. 2003. Ranking metapopulation extinction risk: from patterns in data to conservation management decisions. Ecological Applications 13:990-998.

Figueira, W. F. 2009. Connectivity or demography: defining sources and sinks in coral reef fish metapopulations. Ecological Modelling 220:1126-1137.

Gaines, S. D., C. White, M. H. Carr, and S. R. Palumbi. 2010. Designing marine reserve networks for both conservation and fisheries management. Proceedings of the National Academy of Sciences USA 107:18286-18293.

Gaylord, B., and S. D. Gaines. 2000. Temperature or transport? Range limits in marine species mediated solely by flow. American Naturalist 155:769-789.

Hamilton, S. L., J. Regetz, and R. R. Warner. 2008. Postsettlement survival linked to larval life in a marine fish. Proceedings of the National Academy of Sciences USA 105:1561-1566.

Hastings, A., and L. W. Botsford. 2006. Persistence of spatial populations depends on returning home. Proceedings of the National Academy of Sciences USA 103:6067-6072.

Higgins, S. I., and D. M. Richardson. 1999. Predicting plant migration rates in a changing world: the role of long-distance dispersal. American Naturalist 153:464-475.

Hixon, M. A., S. W. Pacala, and S. A. Sandin. 2002. Population regulation: historical context and contemporary challenges of open vs. closed systems. Ecology 83:1490-1508.

James, M. K., P. R. Armsworth, L. B. Mason, and L. Bode. 2002. The structure of reef fish metapopulations: modelling larval dispersal and retention patterns. Proceedings of the Royal Society B 269:2079-2086.
Jones, G. P., G. R. Almany, G. R. Russ, P. F. Sale, R. S. Steneck, M. J. H. Oppen, and B. L. Willis. 2009. Larval retention and connectivity among populations of corals and reef fishes: history, advances and challenges. Coral Reefs 28:307-325.

Jones, G. P., M. J. Milicich, M. J. Emslie, and C. Lunow. 1999. Self-recruitment in a coral reef fish population. Nature 402:802-804.

Jones, G. P., S. Planes, and S. R. Thorrold. 2005. Coral reef fish larvae settle close to home. Current Biology 15:1314-1318.

Kaplan, D. M., L. W. Botsford, M. R. O'Farrell, S. D. Gaines, and S. Jorgensen. 2009. Model-based assessment of persistence in proposed marine protected area designs. Ecological Applications 19:433-448.

Kinlan, B. P., S. D. Gaines, and S. E. Lester. 2005. Propagule dispersal and the scales of marine community process. Diversity and Distributions 11:139-148.

Koenig, W. D., D. Van Vuren, and P. N. Hooge. 1996. Detectability, philopatry, and the distribution of dispersal distances in vertebrates. Trends in Ecology and Evolution 11:514-517.

Kritzer, J. P., and P. F. Sale, editors. 2006. Marine metapopulations. Elsevier Academic, New York, New York, USA.

Leibold, M. A., M. Holyoak, N. Mouquet, P. Amarasekare, J. M. Chase, M. F. Hoopes, R. D. Holt, J. B. Shurin, R. Law, D. Tilman, M. Loreau, and A. Gonzalez. 2004. The metacommunity concept: a framework for multi-scale community ecology. Ecology Letters 7:601-613.

Leis, J. M., U. Siebeck, and D. L. Dixson. 2011. How Nemo finds home: the neuroecology of dispersal and of population connectivity in larvae of marine fishes. Integrative and Comparative Biology 51:826-843.

Lenormand, T. 2002. Gene flow and the limits to natural selection. Trends in Ecology and Evolution 17:183-189.

Levin, L. A. 2006. Recent progress in understanding larval dispersal: new directions and digressions. Integrative and Comparative Biology 46:282-297.

Levin, S. A. 1992. The problem of pattern and scale in ecology. Ecology 73:1943-1967.

Lockwood, D. R., A. Hastings, and L. W. Botsford. 2002. The effects of dispersal patterns on marine reserves: Does the tail wag the dog? Theoretical Population Biology 61:297-309.

Marshall, D. J., K. Monro, M. Bode, M. J. Keough, and S. Swearer. 2010. Phenotype-environment mismatches reduce connectivity in the sea. Ecology Letters 13:128-140.

Moilanen, A., and I. Hanski. 2006. Connectivity and metapopulation dynamics in highly fragmented landscapes. Pages 44-71 in K. R. Crooks, editor. Connectivity conservation. Cambridge University Press, Cambridge, UK.

Mora, C., and P. F. Sale. 2002. Are populations of coral reef fish open or closed? Trends in Ecology and Evolution 17:422-428.

Palmer, M. A., J. D. Allan, and C. A. Butman. 1996. Dispersal as a regional process affecting the local dynamics of marine and stream benthic invertebrates. Trends in Ecology and Evolution 11:322-326.

Paris, C. B., and R. K. Cowen. 2004. Direct evidence of a biophysical retention mechanism for coral reef fish larvae. Limnology and Oceanography 49:1964-1979.

Pinsky, M., H. R. Montes, Jr., and S. R. Palumbi. 2010. Using isolation by distance and effective density to estimate dispersal scales in anemonefish. Evolution 64:2688-2700.

Planes, S., G. P. Jones, and S. R. Thorrold. 2009. Larval dispersal connects fish populations in a network of marine protected areas. Proceedings of the National Academy of Sciences USA 106:5693-5697.

Puebla, O., E. Bermingham, and F. Guichard. 2009. Estimating dispersal from genetic isolation by distance in a coral reef fish (Hypoplectrus puella). Ecology 90:3087-3098.

Purkis, S. J., K. E. Kohler, B. M. Riegl, and S. O. Rohmann. 2007. The statistics of natural shapes in modern coral reef landscapes. Journal of Geology 115:493-508. 
Roberts, C. M. 1997. Connectivity and management of Caribbean coral reefs. Science 278:1454-1457.

Robertson, D. R. 2001. Population maintenance among tropical reef fishes: inferences from small-island endemics. Proceedings of the National Academy of Sciences USA 98:5667-5670.

Saenz-Agudelo, P., G. P. Jones, S. R. Thorrold, and S. Planes. 2011. Connectivity dominates larval replenishment in a coastal reef fish metapopulation. Proceedings of the Royal Society B 278:2954-2961.

Saunders, D. A., R. J. Hobbs, and C. R. Margules. 1991. Biological consequences of ecosystem fragmentation: a review. Conservation Biology 5:18-32.

Shanks, A. L. 2009. Pelagic larval duration and dispersal distance revisited. Biological Bulletin 216:373-385.

Shima, J. S., E. G. Noonburg, and N. E. Phillips. 2010. Life history and matrix heterogeneity interact to shape metapopulation connectivity in spatially structured environments. Ecology 91:1215-1224.

Siegel, D. A., B. P. Kinlan, and S. D. Gaines. 2003. Lagrangian descriptions of marine larval dispersion. Marine Ecology Progress Series 260:83-96.

Siegel, D. A., S. Mitarai, C. J. Costello, S. D. Gaines, B. E. Kendall, R. R. Warner, and K. B. Winters. 2008. The stochastic nature of larval connectivity among nearshore marine populations. Proceedings of the National Academy of Sciences 105:89748979.

Skellam, J. G. 1951. Random dispersal in theoretical populations. Biometrika 38:196-218.

Swearer, S. E., J. E. Caselle, D. W. Lea, and R. R. Warner. 1999. Larval retention and recruitment in an island population of a coral-reef fish. Nature 402:799-802.

Taylor, M. S., and M. E. Hellberg. 2003. Genetic evidence for local retention of pelagic larvae in a Caribbean reef fish. Science 299:107-109.

Thomas, C. D., and W. E. Kunin. 1999. The spatial structure of populations. Journal of Animal Ecology 68:647-657.
Wabnitz, C. C., S. Andréfouët, and F. E. Muller-Karger. 2010. Measuring progress toward global marine conservation targets. Frontiers in Ecology and the Environment 8:124129.

Waples, R. S. 1998. Separating the wheat from the chaff: patterns of genetic differentiation in high gene flow species. Journal of Heredity 89:438-450.

Waples, R. S., and O. Gaggiotti. 2006. What is a population? An empirical evaluation of some genetic methods for identifying the number of gene pools and their degree of connectivity. Molecular Ecology 15:1419-1439.

Warner, R. R., and R. K. Cowen. 2002. Local retention of production in marine populations: evidence, mechanisms, and consequences. Bulletin of Marine Science 70:S245-S249.

Watson, J. R., S. Mitarai, D. A. Siegel, J. E. Caselle, C. Dong, and J. C. McWilliams. 2010. Realized and potential larval connectivity in the Southern California Bight. Marine Ecology Progress Series 401:31-48.

Watson, J. R., D. A. Siegel, B. E. Kendall, S. Mitarai, A. Rassweiller, and S. D. Gaines. 2011. Identifying critical regions in small-world marine metapopulations. Proceedings of the National Academy of Sciences USA 108:E907-E913.

White, J. W., L. W. Botsford, M. L. Baskett, L. A. K. Barnett, R. J. Barr, and A. Hastings. 2011. Linking models with monitoring data for assessing performance of no-take marine reserves. Frontiers in Ecology and the Environment 9:390399.

White, J. W., L. W. Botsford, A. Hastings, and J. L. Largier. 2010. Population persistence in marine reserve networks: incorporating spatial heterogeneities in larval dispersal. Marine Ecology Progress Series 398:49-67.

Wiens, J. A. 1989. Spatial scaling in ecology. Functional Ecology 3:385-397.

Wieters, E. A., S. D. Gaines, S. A. Navarrete, C. A. Blanchette, and B. A. Menge. 2008. Scale of dispersal and the biogeography of marine predator-prey interactions. American Naturalist 171:405-417.

\section{Supplemental Material}

\section{Appendix}

Maps of the coral reefs, analysis of openness with advection, and analysis of openness at different grid sizes (Ecological Archives A022-066-A1). 